Full length article

\title{
A finite strip for the vibration analysis of rotating cylindrical shells
}

\author{
Ivo Senjanović*, Ivan Ćatipović, Neven Alujević, Nikola Vladimir, Damjan Čakmak \\ University of Zagreb, Faculty of Mechanical Engineering and Naval Architecture, Zagreb, Croatia
}

\section{A R T I C L E IN F O}

\section{Keywords:}

Cylindrical shell

Vibration

Rotating structures

Natural frequencies

Rotating modes

Finite strip method

\begin{abstract}
A B S T R A C T
In this article, a two-node finite strip with eight degrees of freedom for the free vibration analysis of pre-stressed rotating cylindrical shells is formulated. The circumferential mode shape profiles are described exactly using trigonometric functions. The axial mode shape profiles are approximated by bar and beam shape functions for membrane and bending displacements, respectively. In this way, a semi-analytical formulation is facilitated so that discretisation is required only in the axial direction. The accuracy and convergence of the developed finite strip are confirmed by comparisons with the analytical results. Excellent agreement is observed both for stationary and rotating shells.
\end{abstract}

\section{Introduction}

Shells are widely used as constructive elements in many engineering structures. Their static and dynamic behaviour has been an important topic in structural design for a long time [1-3]. As a result, the theory of shells and plates has been covered in a systematic manner in a number of books [4-11]. For example, an instructive approach to thin shell theory, written in a relatively simple way and adapted to the engineering level for practical usage, is presented in [12]. On the other hand, some authors have covered more specific problems related to the design of shell-like structures $[13,14]$. For example, the general theory and specific discussions regarding shells of revolution exposed to a uniform load can be found in [15]. Such problems are more particular for submarine or aircraft pressure hull designs with pronounced axial symmetry.

In fact, it is often the case that axisymmetric shells rotate around the axis of symmetry [16-22,24-30]. Rotating shells of revolution are found in engineering practice in rotor systems of gas turbine engines, high-speed centrifugal separators, rotating satellite structures, automotive tyres, etc. Rotation makes their dynamic behaviour significantly more complex. One of the first investigations into the vibration of rotating structures was carried out by Bryan [16]. He studied the vibrations of a rotating ring and described the travelling modes phenomenon. These phenomena result from the Coriolis effect, as shown in the example of infinitely long rotating cylindrical shells $[17,18]$, as well as in finite rotating cylinders $[19,20]$. An experimental study on the flexural vibrations of a thin rotating ring is given in [21]. Furthermore, the influence of pre-stress on the free vibrations of rotating cylinders has been studied in [22].

Huang and Soedel [24] used nonlinear strain-displacement relationships [23] to formulate the corresponding set of differential equations of motion for a rotating cylindrical shell. They exactly solved the free and forced vibration problem of a simply supported cylindrical shell by assuming simple sine and cosine displacement functions of the circumferential and axial variables. In this case, formulating the eigenvalue/ eigenvector problem results in a characteristic polynomial of the sixth order. Its solution gives three positive and three negative natural frequencies.

If the shell does not rotate, the polynomial is bicubic. There are three pairs of positive/negative frequencies characterised by the same absolute value. This is physically explained through pairs of backward and forward rotating modes. The two modes of a pair rotate with the same speed in opposite directions and thus superimpose into a stationary mode. The reason why there are three pairs of modes and natural frequencies is that there are three types of dominant modes: bending (radial), longitudinal (axial), and shear (circumferential).

If the shell rotates around its axis with a constant speed, then the polynomial is no longer bicubic. The full sixth order polynomial occurs. The positive and negative natural frequencies have distinct absolute values and so-called frequency veering (bifurcation) happens. This means that the forward and backward rotating modes no longer rotate

\footnotetext{
* Corresponding author.

Email address: ivo.senjanovic@fsb.hr (I. Senjanović)
} 
with the same rotation speed and thus cannot superimpose into the stationary modes (standing waves). As a result, with spinning shells the modes rotate independently. For example, this phenomenon comes about with tyres rolling over the road surface [25-29]. This significantly influences the overall NVH (Noise and Vibration Harshness) characteristics of the vehicle.

It is very common in the literature on the vibration of cylindrical shells, either rotating or stationary, to assume that the two ends of the shell are simply supported [24,30,31]. This type of boundary support is sometimes referred to as a shear diaphragm set of boundary conditions $[11,12]$. The reason why this set of boundary conditions is often used is that it is probably the only one for which a relatively simple solution can be obtained analytically. In other words, mode shapes assumed as appropriate products of trigonometric functions of the circumferential and axial variables usually satisfy both the differential equations and the boundary conditions. This results in a mathematically convenient model.

However, such a model is not necessarily suitable to describe a particular engineering problem. For example, although it is very instructive to investigate the dynamics of rotating tyres assuming simply supported edges of a tyre tread-band [24,30,31], it is difficult to accept, from an engineering point of view, that the tyre sidewall is infinitely stiff in radial or tangential directions $[28,29]$.

In the case of cylindrical shells with boundary conditions other than simply supported ones, the mathematics becomes significantly complicated. Closed-form solutions are now difficult to obtain. A number of investigations have been undertaken to tackle this problem [33-39]. One of the solutions was obtained by assuming the shell displacement field as a product of Fourier series in the axial direction, and trigonometric functions in the circumferential direction [36]. This procedure has been recently extended to rotating cylindrical shells [37]. The problem of the free vibration of a rotating cylindrical shell having arbitrary boundary conditions can also be solved by employing the Rayleigh-Ritz method. Such a solution, using characteristic orthogonal polynomials for displacement variations along the axial direction, can be found in [38].

A complete analytical solution for free vibrations of a rotating cylindrical shell with arbitrary boundary conditions has recently been offered in [39]. The equations of motion in [39] are based on the strain-displacement relationships of Hermann and Armenakas [23]. Circumferential tension due to internal pressure or centrifugal forces, as well as an elastic foundation in both radial and circumferential directions, is taken into account. The circumferential mode shape profiles are described by trigonometric functions. The axial profiles are assumed as a sum of eight weighted exponential functions. Three differential equations of motion for an assumed circumferential mode number lead to a frequency equation in the form of a bi-quartic polynomial. Eight cases of the four different combinations of the polynomial roots (real, imaginary and complex) were identified. Hence, the mode shape axial profiles are described in terms of trigonometric functions, hyperbolic functions and their products. The application of the analytical solution was illustrated in the case of a cylindrical shell with free-free boundary conditions, and excellent agreement with the experimentally obtained results was confirmed. The principal advantage of the analytical procedure [39] is very high accuracy confirmed by experiments.

However, the procedure requires the discovery of a proper case among the eight types of axial mode shapes for which a solution can be found even for a single cylindrical shell. If a shell structure consists of $n$ cylindrical shells of different particulars, the number of combinations to find the proper one is $8^{n}$. Hence, although numerical examples solved in this way may be very useful as benchmarks for the evaluation of various numerical methods, the analytical procedure is not quite suitable for practical use.
In practical situations, the problem of the vibration of cylindrical shells with boundary conditions other than simply supported ones could be solved by using the finite element method (FEM). In fact, complex built-up shell structures, which may be approximated by a number of connected cylindrical or other types of shells, could be conveniently tackled by the finite element method. For this purpose, special shell finite elements based on the waveguide finite element method would be suitable. Such finite element analyses have so far been used to tackle stationary shell structures $[43,44]$. Alternatively, the homogeneity of the cylinder around the circumference and along the axis has been exploited to post-process the FE model of a small rectangular segment of the cylinder using periodic structure theory to obtain the wave characteristics of a cylinder [45]. Since there is an integer number of wavelengths around the circumference of a closed circular cylinder, one of the integrals in the inverse Fourier transform becomes a simple summation, whereas the other can be resolved analytically using contour integration and the residue theorem [45].

Although the semi-analytical waveguide approaches of [43-45] may be very convenient and useful for a variety of problems, none of the semi-analytical finite element formulations developed so far a) allow for considering the typical effects of rotation (rotating modes and frequency veering) or b) can take into account the effects of pre-stress due to possible pressurisation and/or centrifugal forces. Therefore, the state-of-the-art in the considered field motivates further developments of finite elements especially tailored to model rotating and pre-stressed cylindrical shells.

One of the very effective numerical methods which reduces the two-dimensional problem into a one-dimensional one in the case of simply supported two opposite edges or shells of revolution is the finite strip method (FSM) [46]. Due to this advantage, the method is widely used in the structural analysis of engineering structures. Some recent publications on different problems are included in the reference list [47-55]. All the articles are published in the Thin-Walled Structures journal as a major forum for the development of the finite strip method.

In this paper, a new finite strip for modelling pre-stressed rotating cylindrical shells is formulated. The energy approach is used with the strain-displacement relationships given in $[23,24]$ to develop and validate such a special finite strip [46]. The strip element is deliberately made quite simple, including two nodes and eight degrees of freedom (d.o.f.). Its reliability is checked in a number of numerical examples by comparing the numerical results with the analytical ones. Excellent agreement is observed for all the boundary conditions considered.

\section{Strain and kinetic energy of a rotating cylindrical shell}

A thin cylindrical shell rotating around its axis of symmetry with constant angular speed $\Omega$ is shown schematically in Fig. 1 . The shell dimensions are the following: $L$ is the length, $a$ is the radius, and $h$ is the thickness. The shell mid-surface is defined in the cylindrical coordinate system, where $x$ and ? are the axial and angular coordinates, respectively. The displacement of a point $P$ on the mid-surface, whose position is defined by $x$ and ?, is specified by the axial, tangential and radial displacement components $u, v$ and $w$, respectively, as shown in Fig. 1.

The problem of shell vibration can be analysed by directly solving differential equations of motion or by minimising the total energy with assumed mode shapes, i.e. by applying the Rayleigh-Ritz method. The equations of motion can be derived by considering the equilibrium of the internal and external forces on an infinitesimally small element [12]. Differential equations of motion can also be obtained by minimisation of the total energy with respect to displacements, i.e. by applying Hamilton's principle. 

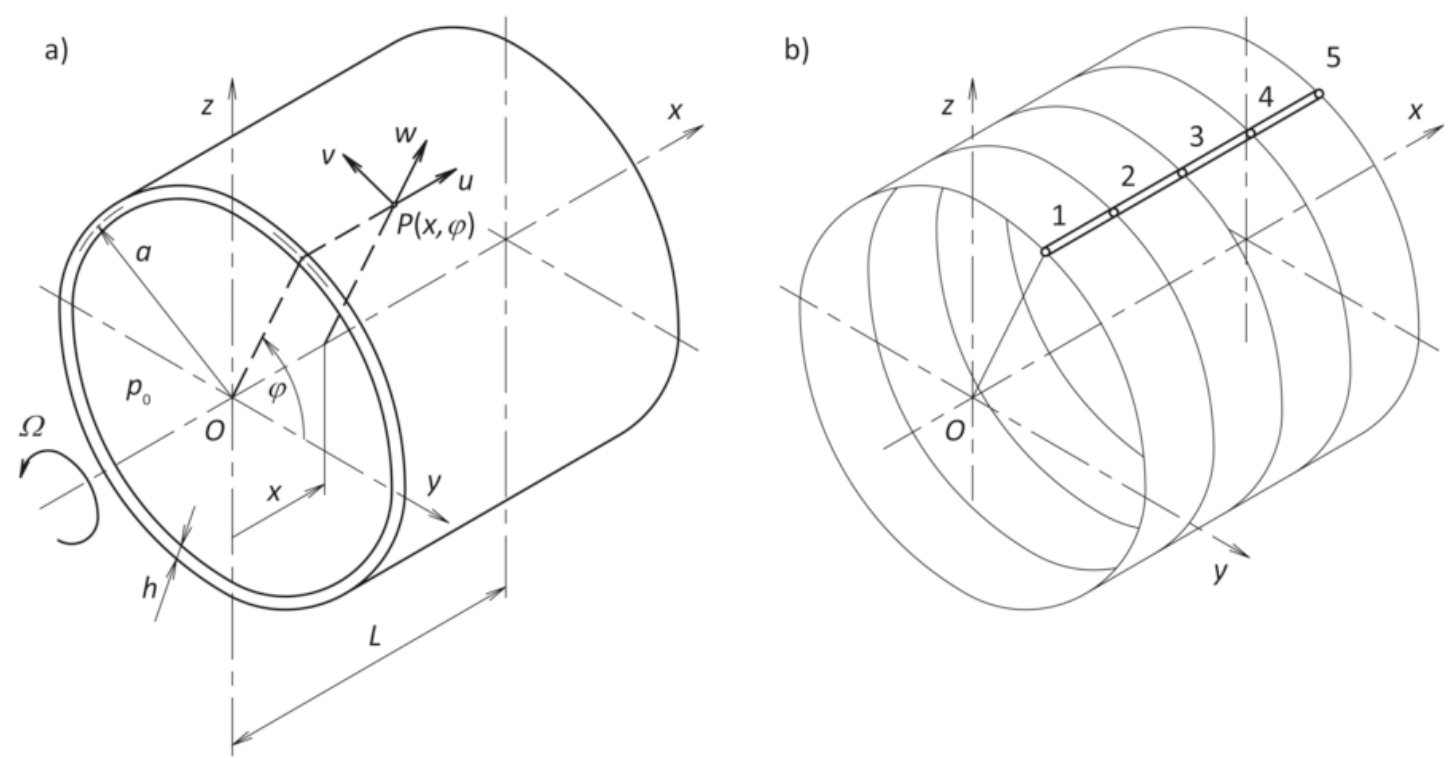

Fig. 1. Rotating cylindrical shell with: a) the main particulars and displacements, b) finite strips with nodes.

In the case of a rotating cylindrical shell, the latter approach is more convenient, as shown for example in [38]. Love's simplification [56] allows for the shell strain field to be separated into membrane strains and bending strains [24]

$\tilde{\varepsilon}_{x}=\varepsilon_{x}+z \kappa_{x}, \quad \tilde{\varepsilon}_{\phi}=\varepsilon_{\phi}+z \kappa_{\phi} \quad \tilde{\varepsilon}_{x \phi}=\varepsilon_{x \phi}+z \kappa_{x \phi}$,

where $\varepsilon_{i}$ are the membrane strains, $\kappa_{i}$ denote the change in curvature due to the shell bending, and $z$ is the distance of a shell layer from the reference mid-surface. According to [24], the membrane strains can be written in the following form:

$$
\begin{array}{r}
\varepsilon_{x}=\frac{\partial u}{\partial x}+\frac{1}{2}\left[\left(\frac{\partial u}{\partial x}\right)^{2}+\left(\frac{\partial v}{\partial x}\right)^{2}+\left(\frac{\partial w}{\partial x}\right)^{2}\right]+\frac{1}{E h}(N \\
\varepsilon_{\phi}=\frac{1}{a}\left(w+\frac{\partial v}{\partial \phi}\right)+\frac{1}{2 a^{2}}\left[\left(\frac{\partial u}{\partial \phi}\right)^{2}+\left(w+\frac{\partial v}{\partial \phi}\right)^{2}+\left(\frac{\partial w}{\partial \phi}-v\right)^{2}\right. \\
\varepsilon_{x \phi}=\frac{1}{a} \frac{\partial u}{\partial \phi}+\frac{\partial v}{\partial x},
\end{array}
$$

whereas the bending strain components are given by:

$$
\begin{gathered}
\kappa_{x}=-\frac{\partial^{2} w}{\partial x^{2}} \\
\kappa_{\phi}=\frac{1}{a^{2}}\left(\frac{\partial v}{\partial \phi}-\frac{\partial^{2} w}{\partial \phi^{2}}\right) \\
\kappa_{x \phi}=\frac{1}{a}\left(\frac{\partial v}{\partial x}-2 \frac{\partial^{2} w}{\partial x \partial \phi}\right),
\end{gathered}
$$

where $N_{x}$ and $N_{\text {? }}$ are the initial tension forces which can be caused by either the centrifugal force, which depends on rotational speed, $\Omega$, or internal pressure, $p_{0}$, or both

$N_{x}=\frac{1}{2} a p_{0}, \quad N_{\phi}=\rho h a^{2} \Omega^{2}+a p_{0}$.

In fact, the nonlinear (quadratic) terms in the first two equations of Eq. (2) are introduced in order to capture the geometric stiffness effect primarily due to these initial tensions.

The three stress components in a shell layer are defined according to the 2D Hooke's law

$$
\begin{aligned}
\tilde{\sigma}_{x} & =\frac{E}{1-v^{2}}\left(\widetilde{\varepsilon}_{x}+v \widetilde{\varepsilon}_{\varphi}\right), \quad \tilde{\sigma}_{\phi} \\
& =\frac{E}{1-v^{2}}\left(\widetilde{\varepsilon}_{\varphi}+v \widetilde{\varepsilon}_{x}\right), \quad \sigma_{x \varphi} \\
& =\frac{E}{2(1+v)} \widetilde{\varepsilon}_{x \varphi},
\end{aligned}
$$

where $E$ is Young's modulus and $v$ is Poisson's ratio. The strain energy stored in an infinitesimal shell element of thickness $h$ is

$d E_{s}=\frac{1}{2} \int_{-\frac{h}{2}}^{\frac{h}{2}}\left(\widetilde{\sigma}_{x} \widetilde{\varepsilon}_{x}+\widetilde{\sigma}_{\phi} \widetilde{\varepsilon}_{\phi}+\widetilde{\sigma}_{x \phi} \widetilde{\varepsilon}_{x \phi}\right) \mathrm{d} z \mathrm{~d} A$.

Substituting Eqs. (1) and (5) into (6) and taking into account that the infinitesimal shell mid-surface $\mathrm{d} A=a \mathrm{~d} x \mathrm{~d} \phi$ yields

$d E_{s}=\frac{a}{2}\left\{K\left[\varepsilon_{x}^{2}+\varepsilon_{\phi}^{2}+2 v \varepsilon_{x} \varepsilon_{\phi}+\frac{1}{2}(1-v) \varepsilon_{x \phi}^{2}\right]+D\left[\kappa_{x}^{2}+\kappa_{\phi}^{2}\right.\right.$

where

$K=\frac{E h}{1-v^{2}}, \quad D=\frac{E h^{3}}{12\left(1-v^{2}\right)}$

are the membrane stiffness and bending stiffness, respectively. Hence, the membrane and bending strain energies are uncoupled in (7) since functions $\varepsilon_{i}$ and $z \kappa_{j}$ are orthogonal within the shell thickness domain. Furthermore, by substituting Eqs. (2) and (3) into Eq. (7) one obtains the expression for the strain energy of an infinitesimal shell element in terms of derivatives of displacements of different orders of magnitude: $\delta_{0}^{0}, \delta_{1}^{1}, \delta_{2}^{2}, \delta_{3}^{3}, \delta_{4}^{4}$. The 3 rd and 4 th order terms can be ignored as small quantities of a higher order. On the other hand, the zero and the 1st order terms are static terms and can also be omitted since the shell vibrates around the static equilibrium. As a result, the strain energy is reduced to the form

$\mathrm{d} E_{s}=F_{s}(x, \phi, t) \mathrm{d} x \mathrm{~d} \phi$, 
where the strain energy density function is given by

$F_{S}(x, \phi, t)=a\left\{\frac{1}{2}\left(K+N_{x}\right)\left(\frac{\partial u}{\partial x}\right)^{2}+\frac{1}{2 a^{2}}\left[\frac{1}{2}(1-v) K+N_{\phi}\right]\right.$

The kinetic energy of an infinitesimal shell element is given by

$d E_{k}=F_{k}(x, \phi, t) \mathrm{d} x \mathrm{~d} \phi$,

where the kinetic energy density function $F_{k}$ is a product of the specific mass per unit area and the total velocity squared. The total velocity consists of the velocity components in the axial, circumferential and radial directions. It also includes contributions due to the shell rotation, i.e.

$$
\begin{aligned}
F_{k}(x, \phi, t)=\frac{1}{2} a \rho h\left\{\left(\frac{\partial u}{\partial t}\right)^{2}+\right. & {\left[\frac{\partial v}{\partial t}+(a+w) \Omega\right]^{2} } \\
& \left.+\left(\frac{\partial w}{\partial t}-v \Omega\right)^{2}\right\} .
\end{aligned}
$$

\section{Differential equations of motion}

\subsection{General cylindrical shell}

Differential equations of motion are obtained by applying Hamilton's principle to the total strain (i.e. potential) and kinetic energy [24]. The total energy of a conservative dynamic system reads

$E=\int_{0}^{2 \pi} \int_{0}^{L}\left[F_{s}(x, \phi, t)+F_{k}(x, \phi, t)\right] \mathrm{d} x \mathrm{~d} \phi$,

where the energy density functions $F_{s}$ and $F_{k}$ are given by Eqs. (10) and (12), respectively. The total energy is constant since there is no energy dissipation due to damping and strain and kinetic energy interchange from their maximum to zero value with the phase shift.

As a result, in the considered case the following three partial differential equations are obtained [24]:

$$
\begin{gathered}
D\left(\frac{\partial^{4} w}{\partial x^{4}}+\frac{2}{a^{2}} \frac{\partial^{4} w}{\partial x^{2} \partial \varphi^{2}}+\frac{1}{a^{4}} \frac{\partial^{4} w}{\partial \varphi^{4}}\right)-N_{x} \frac{\partial^{2} w}{\partial x^{2}}-N_{\phi} \frac{1}{a^{2}} \frac{\partial^{2} w}{\partial \varphi^{2}}+\frac{1}{a^{2}}(\boldsymbol{K} \\
+v \frac{K}{a} \frac{\partial u}{\partial x}-\frac{D}{a^{2}} \frac{\partial^{3} v}{\partial x^{2} \partial \varphi}-\frac{D}{a^{4}} \frac{\partial^{3} v}{\partial \varphi^{3}}+\frac{1}{a^{2}}\left(K+2 N_{\varphi}\right) \frac{\partial v}{\partial \varphi} \\
+\rho h \frac{\partial^{2} w}{\partial t^{2}}-\rho h \Omega^{2} w-2 \rho h \Omega \frac{\partial v}{\partial t}=0
\end{gathered}
$$$$
-\left(K+N_{x}\right) \frac{\partial^{2} u}{\partial x^{2}}-\left[\frac{1}{2}(1-v) K+N_{\phi}\right] \frac{1}{a^{2}} \frac{\partial^{2} u}{\partial \phi^{2}}
$$$$
-\frac{1}{2}(1+v) \frac{K}{a} \frac{\partial^{2} v}{\partial x \partial \phi}-v \frac{K}{a} \frac{\partial w}{\partial x}+\rho h \frac{\partial^{2} u}{\partial t^{2}}=0 \text {, }
$$

$$
\begin{gathered}
-\frac{1}{2}\left[\frac{1}{2}(1-v)\left(\frac{D}{a^{2}}+K\right)+N_{x}\right] \frac{\partial^{2} v}{\partial x^{2}}-\left(\frac{D}{a^{2}}+K+N_{\phi}\right) \frac{1}{a^{2}} \frac{\partial^{2} v}{\partial \phi^{2}}+ \\
-\frac{1}{2}(1+v) \frac{K}{a} \frac{\partial^{2} u}{\partial x \partial \phi}+\frac{D}{a^{2}} \frac{\partial^{3} w}{\partial x^{2} \partial \phi}+\frac{D}{a^{4}} \frac{\partial^{3} w}{\partial \phi^{3}}-\frac{1}{a^{2}}\left(K+2 N_{\phi}\right) \frac{\partial}{\partial} \\
+\rho h \frac{\partial^{2} v}{\partial t^{2}}-\rho h \Omega^{2} v+2 \rho h \Omega \frac{\partial w}{\partial t}=0 .
\end{gathered}
$$

3.2. Cylindrical shell with a closed cross-section and arbitrary boundary conditions

For a cylindrical shell with a closed cross-section and arbitrary boundary conditions at the two ends (at $x=0$ and $x=L$, where $L$ is the length of the shell), the three displacement components can be assumed in the form

$$
\begin{gathered}
u(x, \phi, t)=U(x) \cos (n \phi+\omega t) \\
v(x, \phi, t)=V(x) \sin (n \phi+\omega t) \\
w(x, \phi, t)=W(x) \cos (n \phi+\omega t),
\end{gathered}
$$

where $\omega$ is a natural frequency. Note that in Eq. (17) the variables are separated in such a way that displacements are assumed as products of their axial and circumferential profiles. Each profile is a function of either the axial or circumferential coordinate only. The circumferential profiles are described through an appropriate set of trigonometric functions, whereas the axial profiles are provisionally left open as functions of the axial variable $U(x), V(x)$, and $W(x)$. Further to this, note that the arguments of the trigonometric functions of the circumferential coordinate allow for the modal shapes to rotate around the cylinder axis.

Substituting (17) into (16), the system of partial differential equations is reduced to the system of ordinary differential equations

$$
\begin{gathered}
D W^{\prime \prime \prime \prime}-\left(2 \frac{n}{a^{2}} D+N_{x}\right) W^{\prime \prime}+\frac{1}{a^{2}}\left[\frac{n^{4}}{a^{2}} D+K+\left(1+n^{2}\right) N_{\phi}\right] W \\
+\frac{v}{a} K U^{\prime}-\frac{n}{a^{2}} D V^{\prime \prime}+\frac{n}{a^{2}}\left(\frac{n}{a^{2}} D+K+2 N_{\phi}\right) V \\
-\rho h\left(\omega^{2}+\Omega^{2}\right) W-2 \rho h \Omega \omega V=0 \\
-\left(K+N_{x}\right) U^{\prime \prime}+\frac{n^{2}}{a^{2}}\left[\frac{1}{2}(1-v) K+N_{\phi}\right] U \\
-\frac{1}{2}(1+v) \frac{n}{a} K V^{\prime}-\frac{v}{a} K W^{\prime}-\rho h \omega^{2} U=0 \\
-\left[\frac{1}{2}(1-v)\left(\frac{D}{a^{2}}+K\right)+N_{x}\right] V^{\prime \prime}+\frac{1}{a^{2}}\left[n^{2}\left(\frac{D}{a^{2}}+K\right)+\left(1+n^{2}\right.\right. \\
+\frac{1}{2}(1+v) \frac{n}{a} K U^{\prime}-\frac{n}{a^{2}} D W^{\prime \prime}+\frac{n}{a^{2}}\left(\frac{n}{a^{2}} D+K+2 N_{\phi}\right) W \\
-\rho h\left(\omega^{2}+\Omega^{2}\right) V-2 \rho h \Omega \omega W=0
\end{gathered}
$$

indicating that the assumed displacement circumferential profiles satisfy the equations of motion. The prime designates a spatial derivative by $x$.

The system of differential Eqs. (18), (19) and (20) has been solved in a general form in [39], assuming the displacement functions in the exponential form

$$
\begin{array}{rlr}
U(x) & =U_{0} \mathrm{e}^{\alpha x / a}, \quad V(x) \\
& =V_{0} \mathrm{e}^{\alpha x / a}, \quad W(x) \\
& =W_{0} \mathrm{e}^{\alpha x / a},
\end{array}
$$

where $\alpha$ is a complex parameter. Substituting (21) into Eqs. (18), (19) and (20), the eigenvalue problem is formulated

$[\mathrm{K}(\omega)]\left\{\begin{array}{c}U_{0} \\ V_{0} \\ W_{0}\end{array}\right\}=\{0\}$,

where $[\mathrm{K}(\omega)]$ is a symmetric dynamic stiffness matrix. Its determinant is expanded into an eighth-order polynomial with even exponents (bi- 
quartic polynomial), with an unknown parameter $\alpha$

$$
\sum_{i=0}^{4} A_{2 i} \alpha^{2 i}=0
$$

As a result, each of the displacement functions is expressed by eight independent exponential functions with eight complex constants [39].

The above analytical solution is rather involved since eight types of complex roots of Eq. (23) are possible depending on the shell particulars. Nevertheless, the analytical solutions according to [39] are used in this paper as a benchmark to cross-check the results obtained by the present numerical method, and thus to validate the newly formulated finite strip.

\subsection{Simply supported cylindrical shell}

As already mentioned in the introduction, in this special case harmonic functions can be assumed for the axial profiles of the displacement components $U(x), V(x)$, and $W(x)$. Apart from satisfying the equations of motion, such axial profiles also satisfy the corresponding shear diaphragm boundary conditions $[12,24]$

$$
\begin{aligned}
U(x) & =A \cos \frac{m \pi x}{m \frac{L}{L}} \\
V(x) & =B \sin \frac{m}{m} \\
W(x) & =C \sin \frac{m \pi x}{L} .
\end{aligned}
$$

The parameter $m$ denotes the mode number which is the number of half-waves along the length of the shell. Substituting Eq. (24) into (14)-(16) results in the following eigenvalue problem (see also [24]):

$\left[\begin{array}{ccc}\omega^{2}-a_{11} & a_{12} & a_{13} \\ a_{21} & \omega^{2}-a_{22} & 2 \Omega \omega-a_{23} \\ a_{31} & 2 \Omega \omega-a_{32} & \omega^{2}-a_{33}\end{array}\right]\left\{\begin{array}{l}A \\ B \\ C\end{array}\right\}=\{0\}$,

where

$$
\begin{array}{r}
a_{11}=\frac{1}{\rho h}\left\{\left(K+N_{x}\right)\left(\frac{m \pi}{L}\right)^{2}+\frac{n^{2}}{a^{2}}\left[\frac{1}{2}(1-v) \boldsymbol{F}\right.\right. \\
a_{22}=\frac{1}{\rho h}\left\{\left[\frac{1}{2}(1-v)\left(\frac{D}{a^{2}}+K\right)+N_{x}\right]\left(\frac{m \pi}{L}\right)^{2}+\frac{1}{a^{2}}\left[n ^ { 2 } \left(\frac{D}{a^{2}}+\right.\right.\right. \\
a_{33}=\frac{1}{\rho h}\left\{D\left(\frac{m \pi}{L}\right)^{4}+\left(2 \frac{n^{2}}{a^{2}} D+N_{x}\right)\left(\frac{m \pi}{L}\right)^{2}+\frac{1}{a^{2}}\left[\frac{n^{4}}{a^{2}} D+\right.\right. \\
a_{12}=\frac{1}{2 \rho h}(1+v) \frac{n}{a} K \frac{m \pi}{L} \\
a_{13}=\frac{1}{\rho h} \frac{v}{a} K \frac{m \pi}{L} \\
a_{23}=\frac{1}{\rho h}\left[\frac{n}{a^{2}} D\left(\frac{m \pi}{L}\right)^{2}+\frac{n}{a^{2}}\left(\frac{n^{2}}{a^{2}} D+K+\right.\right. \\
a_{21}=a_{12}, \quad a_{31}=a_{13}, \quad a_{32}=a_{23}
\end{array}
$$

The determinant of the dynamic stiffness matrix in (25) is either a full sixth-order polynomial for rotating shells, or a bicubic polynomial for stationary shells.

\section{The finite strip}

\subsection{Stiffness matrix}

The cylindrical shell is modelled by a number of finite strips, as shown in Fig. 1.b). Due to the harmonic variation of the displacement field in the circumferential direction, the dynamic behaviour of the finite strip becomes representative by its axial generatrix which behaves like a sophisticated beam.

In this section the properties of the finite strip of a rotating cylindrical shell are developed by employing the energy approach. The stiffness matrix is obtained using the strip strain energy (9)

$E_{s}=\int_{0}^{2 \pi} \int_{0}^{l} F_{s}(x, \phi, t) \mathrm{d} x \mathrm{~d} \phi$

where $F_{s}$ is specified by (10), and $l$ is the strip width. Substituting the assumed displacement functions (17) into (10) and integrating Eq. (27) over the circumference?, the strain energy is obtained in the form

$E_{s}=\int_{0}^{l}\left[\frac{1}{2} a_{1}\left(U^{\prime}\right)^{2}+\frac{1}{2} a_{2} U^{2}+\frac{1}{2} a_{3}\left(V^{\prime}\right)^{2}+\frac{1}{2} a_{4} V^{2}+a_{5} U^{\prime} V\right.$

where

$$
\begin{gathered}
a_{1}=\pi a\left(K+N_{x}\right) \\
a_{2}=\pi \frac{n^{2}}{a}\left[\frac{1}{2}(1-v) K+N_{\varphi}\right] \\
a_{3}=\pi a\left[\frac{1}{2}(1-v)\left(\frac{D}{a^{2}}+K\right)+N_{x}\right] \\
a_{4}=\frac{\pi}{a}\left[n^{2}\left(\frac{D}{a^{2}}+K\right)+\left(1+n^{2}\right) N_{\varphi}\right] \\
a_{5}=\pi v n K \\
a_{6}=-\frac{\pi}{2}(1-v) n K
\end{gathered}
$$

and

$$
\begin{gathered}
b_{1}=\pi a D \\
b_{2}=\pi a\left(2(1-v) \frac{n^{2}}{a^{2}} D+N_{x}\right) \\
b_{3}=\frac{\pi}{a}\left[\frac{n^{4}}{a^{2}} D+K+\left(1+n^{2}\right) N_{\phi}\right] \\
b_{4}=-v \pi \frac{n^{2}}{a} D \\
b_{5}=v \pi K \\
b_{6}=(1-v) \pi \frac{n}{a} D \\
b_{7}=\pi \frac{n}{a}\left(\frac{n^{2}}{a^{2}} D+K+2 N_{\phi}\right) \\
b_{8}=-v \pi \frac{n}{a} D .
\end{gathered}
$$

The strain energy (28) is time invariant. This is due to the fact that the rotating modes are characterised by fixed profiles which only rotate along the circumference.

A relatively simple two nodal line finite strip is introduced next. Amplitudes of the displacement components, Eq. (17), are approximated by the following interpolation functions

$$
\begin{aligned}
U(x) & =\sum_{j=1}^{2} U_{j} g_{j}(x), \quad V(x) \\
& =\sum_{j=1}^{2} V_{j} g_{j}(x), \quad W(x) \\
& =\sum_{j=1}^{4} \Delta_{j} f_{j}(x),
\end{aligned}
$$

where 


$$
\{U\}=\left\{\begin{array}{c}
U_{1} \\
U_{2}
\end{array}\right\}, \quad\{V\}=\left\{\begin{array}{l}
V_{1} \\
V_{2}
\end{array}\right\}, \quad\{\Delta\}=\left\{\begin{array}{c}
W_{1} \\
\Theta_{1} \\
W_{2} \\
\Theta_{2}
\end{array}\right\}
$$

are vectors of the amplitudes of the nodal line displacements, i.e. nodal displacements for short, Fig. 2. For membrane displacements, ordinary polynomial bar shape functions are used. For bending displacements, beam shape functions (Hermitian polynomials) are used:

$$
\begin{gathered}
g_{1}=1-\xi, \quad g_{2}=\xi \\
f_{1}=1-\xi^{2}(3-2 \xi), \quad f_{2}=l \xi(1-\xi)^{2}, \\
f_{3}=\xi^{2}(3-2 \xi), \quad f_{4}=-l \xi^{2}(1-\xi),
\end{gathered}
$$

where $\xi=x / l$ is the dimensionless axial coordinate.

Substituting expressions (31) into (28) and differentiating the strain energy with respect to the nodal displacements, a system of eight algebraic equations is obtained:

$$
\begin{aligned}
& \left\{\frac{\partial E_{s}}{\partial U_{i}}\right\}_{i=1,2}=\left(a_{1}[K]_{1}+a_{2}[K]_{2}\right)\{U\} \\
& +\left(a_{5}[K]_{3}+a_{6}[K]_{3}^{T}\right)\{V\} \\
& +b_{5}[K]_{8}\{\delta\}\left\{\frac{\partial E_{s}}{\partial V_{i}}\right\}_{i=1,2} \\
& =\left(a_{5}[K]_{3}^{T}+a_{6}[K]_{3}\right)\{U\} \\
& +\left(a_{3}[K]_{1}+a_{4}[K]_{2}\right)\{V\} \\
& +\left(b_{6}[K]_{9}+b_{7}[K]_{10}\right. \\
& \left.+b_{8}[K]_{11}\right)\{\delta\}\left\{\frac{\partial E_{s}}{\partial \Delta_{i}}\right\}_{i=1,2,3,4} \\
& =\left(b_{1}[K]_{4}+b_{2}[K]_{5}+b_{3}[K]_{6}\right. \\
& \left.+b_{4}[K]_{7}\right)\{\delta\} \\
& +b_{5}[K]_{8}^{T}\{U\}+\left(b_{6}[K]_{9}^{T}+b_{7}[K]_{10}^{T}\right. \\
& \left.+b_{8}[K]_{11}^{T}\right)\{V\}
\end{aligned}
$$
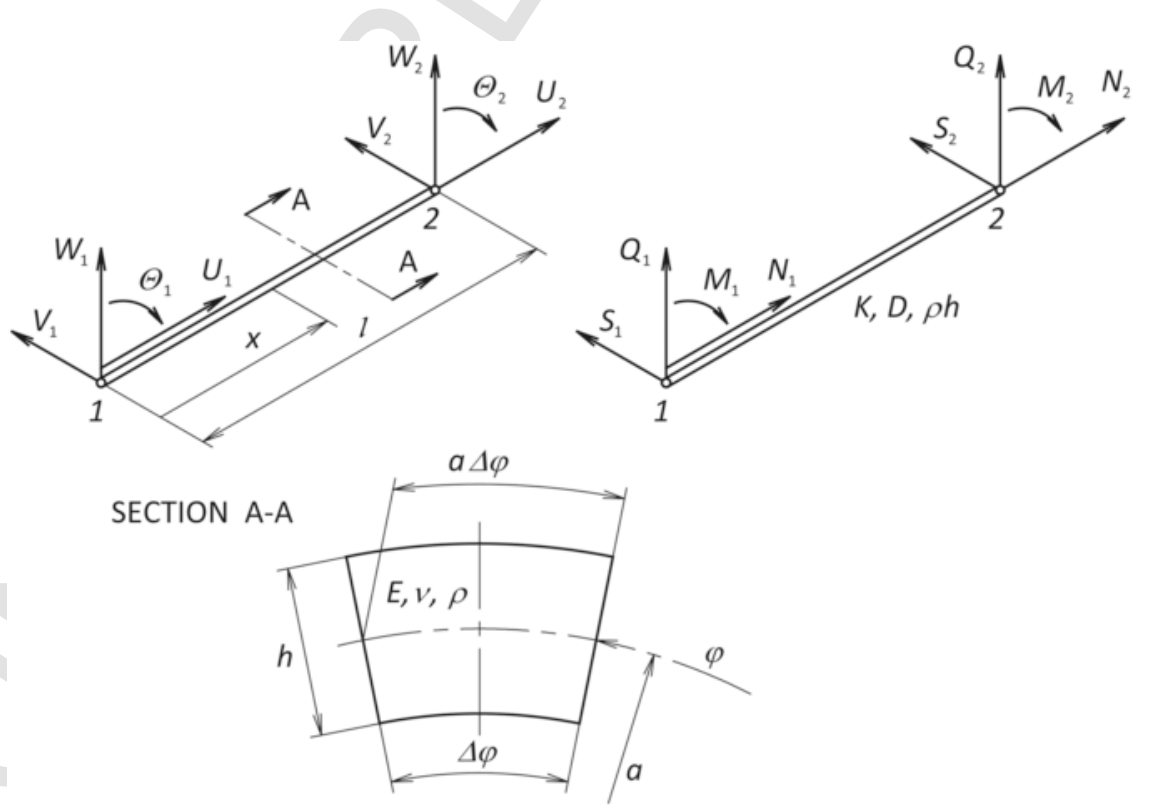

Fig. 2. Nodal displacements and nodal forces of the finite strip and its cross-section. 
Substituting (12) and (17) into (38), one obtains after integration over the circumference?

$$
\begin{aligned}
E_{k}=\pi & a \rho h \int_{0}^{l}\left[\frac{1}{2} \omega^{2} U^{2}+\frac{1}{2}\left(\omega^{2}+\Omega^{2}\right) V^{2}\right. \\
& \left.+\frac{1}{2}\left(\omega^{2}+\Omega^{2}\right) W^{2}+2 \omega \Omega W V+a^{2} \Omega^{2}\right] d x .
\end{aligned}
$$

The kinetic energy (39) is also time invariant, as is the strain energy (28) for the same reason of rotating fixed mode profiles. Furthermore, substituting expressions (31) into (39) and differentiating the kinetic energy with respect to the nodal displacements yields

$$
\begin{gathered}
\left\{\frac{\partial E_{k}}{\partial U_{i}}\right\}_{i=1,2}=\pi a \rho h \omega^{2}[K]_{2}\{U\}, \\
\left\{\frac{\partial E_{k}}{\partial V_{i}}\right\}_{i=1,2}=\pi a \rho h\left(\omega^{2}+\Omega^{2}\right)[K]_{2}\{V\}+2 \pi a \rho h \Omega \omega[K]_{10}\{\delta \\
\left\{\frac{\partial E_{k}}{\partial \Delta_{i}}\right\}_{i=1,2,3,4}=\pi a \rho h\left(\omega^{2}+\Omega^{2}\right)[K]_{6}\{\delta\}+2 \pi a \rho h \Omega \omega[K]_{10}^{T}\{
\end{gathered}
$$

Submatrices $[K]_{i}, i=2,6,10$, are given in Appendix A.

The system of algebraic Eq. (40) can be written in the matrix form

$\frac{\partial E_{k}}{\partial\{\delta\}}=[A]\{\delta\}$,

where $\{\delta\}$ is a vector of nodal displacements, Eq. (36). Matrix [A] can be separated into three matrices with respect to the power of frequency $\omega$

$$
[A]=[B]+\omega[C]+\omega^{2}[M],
$$

which are of the following form:

$$
\begin{gathered}
{[B]=\pi a \rho h \Omega^{2}\left[\begin{array}{ccc}
{[0]} & {[0]} & {[0]} \\
{[0]} & {[K]_{2}} & {[0]} \\
{[0]} & {[0]} & {[K]_{6}}
\end{array}\right]} \\
{[C]=2 \pi a \rho h \Omega\left[\begin{array}{ccc}
{[0]} & {[0]} & {[0]} \\
{[0]} & {[0]} & {[K]_{10}} \\
{[0]} & {[K]_{10}^{T}} & {[0]}
\end{array}\right]} \\
{[M]=\pi a \rho h\left[\begin{array}{ccc}
{[K]_{2}} & {[0]} & {[0]} \\
{[0]} & {[K]_{2}} & {[0]} \\
{[0]} & {[0]} & {[K]_{6}}
\end{array}\right] .}
\end{gathered}
$$

\subsection{Finite strip equation}

It is well known that a stationary linear conservative dynamic system, vibrating freely without external excitation at its natural frequencies, interchanges its vibration energy from a purely potential state, with respect to the static equilibrium as the reference state at its maximum amplitude, $E_{\text {smax }}$, to a purely kinetic state when all vibration amplitudes are zero, $E_{k \max }$ [12]. Hence, the maximum potential energy is equal to the maximum kinetic energy since there is no energy dissipation due to damping. This fact is used in the Rayleigh-Ritz method, assuming the displacement functions in the form of a series in which the coordinate functions individually satisfy the geometric boundary conditions. Since the displacements are approximated, the difference of maximum energies $\Pi=E_{s \max }-E_{k \max }$ is not equal to zero. The relative values of the unknown coefficients of the series, $C_{i}$, as well as the natural frequencies, are determined from the principle of minimum total energy, $\partial \Pi / \partial C_{i}=0$, [57]. If an arbitrary reference state is used for vibration analysis, $\Pi$ is equal to a constant whose derivative vanishes.

The same energy approach is employed in the finite element method and finite strip method. Instead of coordinate functions over the whole structure, shape functions over the finite element and the finite strip are used.

Concerning a finite strip of rotating cylindrical shell strain energy and kinetic energy, Eqs. (28) and (38), respectively, are time invariant and it is not necessary to search for their maximum values as in the case of a stationary shell. However, these energies are not balanced at the finite strip level. Their difference is compensated with the work of nodal forces, $W_{F}$, as an external load. Hence the energy balance reads

$\Pi=E_{s}-E_{k}+W_{F}$,

where $W_{F}=-\langle\delta\rangle\{F\},\langle\delta\rangle$ is a vector of nodal displacements, Eq. (36), and

$$
\langle F\rangle=\{F\}^{T}=\left\langle N_{1} N_{2} S_{1} S_{2} Q_{1} M_{1} Q_{2} M_{2}\right\rangle
$$

is a vector of the corresponding nodal forces, in which $N_{i}$ and $S_{i}, i=$ 1,2 , are tension and in-plane shear forces, while $Q_{i}$ and $M_{i}, i=1,2$, are transverse shear forces and bending moments, respectively, Fig. 2. In an exact solution, the value of $\Pi$, Eq. (46), has to be zero, if free vibrations are analysed with respect to a static reference state, while in an approximated solution it has to be minimum. This is achieved by setting $\partial \Pi / \partial\{\delta\}=\{0\}$, which leads to

$\{F\}=\frac{\partial E_{s}}{\partial\{\delta\}}-\frac{\partial E_{k}}{\partial\{\delta\}}$

since $\partial\langle\delta\rangle / \partial\{\delta\}$ is a unit matrix [I]. Furthermore, taking into account derivatives of the strain and kinetic energy, Eqs. (35) and (41), and relation (42), one obtains the finite strip equation in the following form:

$$
\{F\}=\left([K]-[B]-\omega[C]-\omega^{2}[M]\right)\{\delta\} .
$$

Matrix $[B]$, which depends on $\Omega^{2}$, reduces the element stiffness due to the rotating mass, whereas the geometric stiffness matrix contained in $[\mathrm{K}]$ has the opposite effect. However, the net effect of terms depending on $\Omega^{2}$ is an increase of natural frequencies.

Generally speaking, the accuracy of the FSM analysis is increased if the shell is modelled by a larger number of strips. This is particularly important to properly describe vibration modes with higher axial mode numbers. In order to facilitate a chain-like assembly of the strip equations, nodal displacements and forces in the corresponding vectors (36) and (47) have to be grouped first for the 1st node and then for the 2nd one

$$
\begin{gathered}
\langle\tilde{\delta}\rangle=\left\langle\begin{array}{llllllll}
U_{1} & V_{1} & W_{1} & \Theta_{1} & U_{2} & V_{2} & W_{2} & \Theta_{2}
\end{array}\right\rangle \\
\langle\widetilde{F}\rangle=\left\langle\begin{array}{llllllll}
N_{1} & S_{1} & Q_{1} & M_{1} & N_{2} & S_{2} & Q_{2} & M_{2}
\end{array}\right\rangle .
\end{gathered}
$$

Consequently, the rows and columns of all matrices in Eq. (49) have to be rearranged accordingly. Hence, one may write

$\{\widetilde{F}\}=\left([\widetilde{K}]-[\widetilde{B}]-\omega[\widetilde{C}]-\omega^{2}[\widetilde{M}]\right)\{\widetilde{\delta}\}$.

The coupling between the membrane and bending deformations is very strong and is realised by the stiffness matrix $[K]$, Eq. (37). There is also some weak coupling due to the rotation which is realised through matrix [C], Eq. (44). 


\section{Numerical examples}

\subsection{Simply supported shell}

In order to illustrate the application of the developed finite strip and to evaluate its accuracy, a number of numerical examples considering various boundary conditions are presented. First, a free vibration analysis of a simply supported cylindrical shell with the following geometric particulars and material properties of rubber is carried out using the example from [39]:

$$
\begin{aligned}
L & =0.2 \mathrm{~m}, a \\
& =0.1 \mathrm{~m}, \mathrm{~h} \\
& =0.002 \mathrm{~m}, E \\
& =0.45 \mathrm{GPa}, \quad v \\
& =0.45, \rho \\
& =1452 \mathrm{~kg} / \mathrm{m}^{3} .
\end{aligned}
$$

According to the assumed displacement functions (24), the boundary conditions are: $V(0)=V(L)=W(0)=W(L)=0, N_{x}(0)=N_{x}(L)$ and $M_{x}(0)=M_{x}(L)$. The shell geometry was modelled with 20 equal finite strips and the first $m \times n=8 \times 10=80$ natural modes and the corresponding natural frequencies were determined. The natural frequencies are listed in Table 1, where they are contrasted to the exact values determined according to the procedure described in Section 3.3 [24]. As can be seen in Table 1, excellent agreement of the numerical and analytical results is achieved.

The convergence of the natural frequencies to the analytical values is analysed by increasing the number of finite strips, $N_{e}$. The obtained results are shown in Tables $2-5$ for a set of $m \times n=8 \times 4$ modes. The convergence is from the above and is quite fast.

The bifurcation of natural frequencies due to the angular velocity, $\Omega$, for a few characteristic $m \times n$ natural modes was calculated using 20 strip elements, and is shown in Fig. 3. The same results were obtained analytically. In fact, the analytically obtained bifurcation curves perfectly overlap with the curves plotted using the present finite strip. The values of normalising natural frequency $\omega_{m n}^{0}$ in Fig. 3 are those obtained in the case of $\Omega=0$, Table 1 . For the rotating cylindrical shell, each natural frequency is split into two values, i.e. a forward (lower) and backward (higher) value. In the case of $m \times n=1 \times 1$, the negative value of forward frequency $\omega_{11}$ is obtained for $\Omega / \omega_{11}^{0}>1$. This means that the rotation speed is higher than the speed of the travelling mode. Such a case can also be noticed for mode $m \times n=3 \times 1$. The absolute values of negative natural frequencies are shown in Fig. 3 having in mind the above meaning .

In addition, a parametric analysis of the internal pressure, $p_{0}$, was performed. Fig. 4 shows the increase of natural frequencies due to increased internal pressure. This is a result of an increase in general stiff-

Table 1

\begin{tabular}{|c|c|c|c|c|c|c|c|c|}
\hline $\mathrm{Ne}$ & 1 & 2 & 3 & 4 & 5 & 6 & 7 & 8 \\
\hline 2 & 571.2 & 902.7 & 961.9 & 1034.3 & 1260.3 & 2043.4 & $80,308.0$ & $92,737.2$ \\
\hline 4 & 536.2 & 811.1 & 896.0 & 939.0 & 1039.7 & 1122.2 & 1377.0 & 1522.6 \\
\hline 6 & 529.2 & 795.7 & 873.9 & 927.2 & 991.4 & 1068.5 & 1227.2 & 1432.0 \\
\hline 8 & 526.8 & 790.0 & 864.3 & 913.0 & 972.4 & 1057.7 & 1180.0 & 1354.8 \\
\hline 10 & 525.6 & 787.3 & 859.5 & 905.6 & 961.7 & 1042.9 & 1158.6 & 1315.4 \\
\hline 20 & 524.1 & 783.7 & 852.8 & 894.8 & 946.2 & 1021.9 & 1130.4 & 1276.5 \\
\hline Analytical & 524.0 & 783.0 & 851.1 & 891.7 & 941.4 & 1015.2 & 1122.0 & 1266.3 \\
\hline
\end{tabular}

Natural frequencies of simply supported cylindrical shell, $\omega[\mathrm{Hz}], \Omega=0, p_{0}=0$.

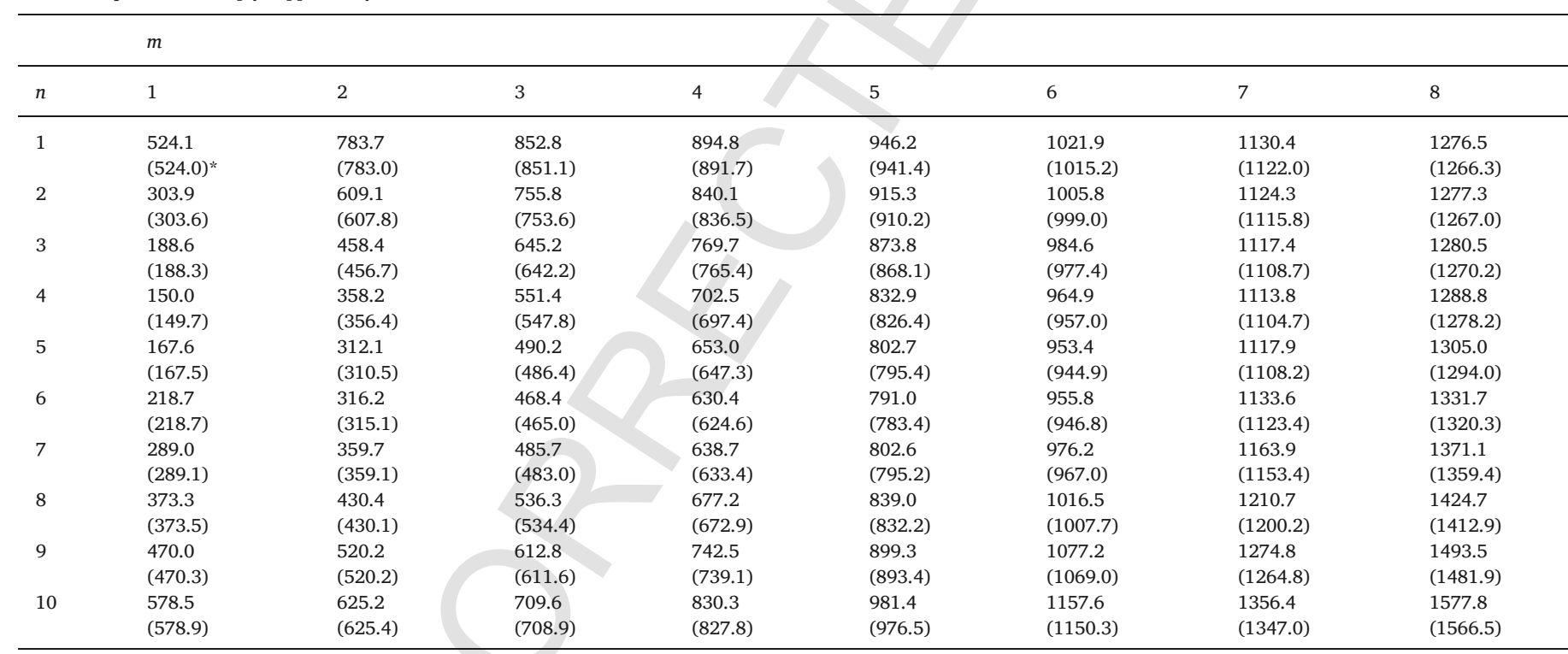

()* - Analytical solution.

Table 2

Convergence of natural frequencies of simply supported cylindrical shell, $\omega[\mathrm{Hz}], \Omega=0, p_{0}=0, n=1$. 
Table 3

Convergence of natural frequencies of simply supported cylindrical shell, $\omega[\mathrm{Hz}], \Omega=0, p_{0}=0, n=2$.

\begin{tabular}{|c|c|c|c|c|c|c|c|c|}
\hline & $m$ & & & & & & & \\
\hline $\mathrm{Ne}$ & 1 & 2 & 3 & 4 & 5 & 6 & 7 & 8 \\
\hline 2 & 357.0 & 910.3 & 981.3 & 1038.5 & 1040.5 & 1586.1 & 3613.4 & $97,489.4$ \\
\hline 4 & 317.4 & 648.3 & 814.3 & 944.9 & 1025.9 & 1040.5 & 1361.4 & 1464.7 \\
\hline 6 & 309.6 & 626.1 & 782.7 & 878.3 & 966.7 & 1040.6 & 1224.2 & 1424.0 \\
\hline 8 & 306.8 & 617.9 & 770.0 & 860.7 & 943.5 & 1040.6 & 1175.1 & 1366.8 \\
\hline 10 & 305.6 & 614.1 & 764.0 & 852.1 & 931.8 & 1027.6 & 1153.0 & 1316.4 \\
\hline 20 & 303.9 & 609.1 & 755.8 & 840.1 & 915.3 & 1005.8 & 1124.3 & 1277.3 \\
\hline Analytical & 303.6 & 607.8 & 753.6 & 836.5 & 910.2 & 999.0 & 1115.8 & 1267.0 \\
\hline
\end{tabular}

Table 4

Convergence of natural frequencies of simply supported cylindrical shell, $\omega[\mathrm{Hz}], \Omega=0, p_{0}=0, n=3$.

\begin{tabular}{|c|c|c|c|c|c|c|c|c|}
\hline & $m$ & & & & & & & \\
\hline $\mathrm{Ne}$ & 1 & 2 & 3 & 4 & 5 & 6 & 7 & 8 \\
\hline 2 & 242.2 & 922.1 & 996.6 & 1046.2 & 1560.8 & 1966.3 & 3486.7 & 3794.0 \\
\hline 4 & 201.7 & 507.9 & 715.7 & 955.3 & 1030.7 & 1178.4 & 1396.3 & 1552.2 \\
\hline 6 & 194.0 & 479.9 & 680.7 & 816.7 & 932.1 & 1093.1 & 1229.1 & 1448.4 \\
\hline 8 & 191.4 & 469.4 & 663.7 & 794.8 & 906.0 & 1025.2 & 1170.0 & 1387.0 \\
\hline 10 & 190.2 & 464.6 & 655.7 & 784.1 & 892.4 & 1008.1 & 1147.3 & 1320.3 \\
\hline 20 & 188.6 & 458.4 & 645.2 & 769.7 & 873.8 & 984.6 & 1117.4 & 1280.5 \\
\hline Analytical & 188.3 & 456.7 & 642.2 & 765.4 & 868.1 & 977.4 & 1108.7 & 1270.2 \\
\hline
\end{tabular}

Table 5

Convergence of natural frequencies of simply supported cylindrical shell, $\omega[\mathrm{Hz}], \Omega=0, p_{0}=0, n=4$.

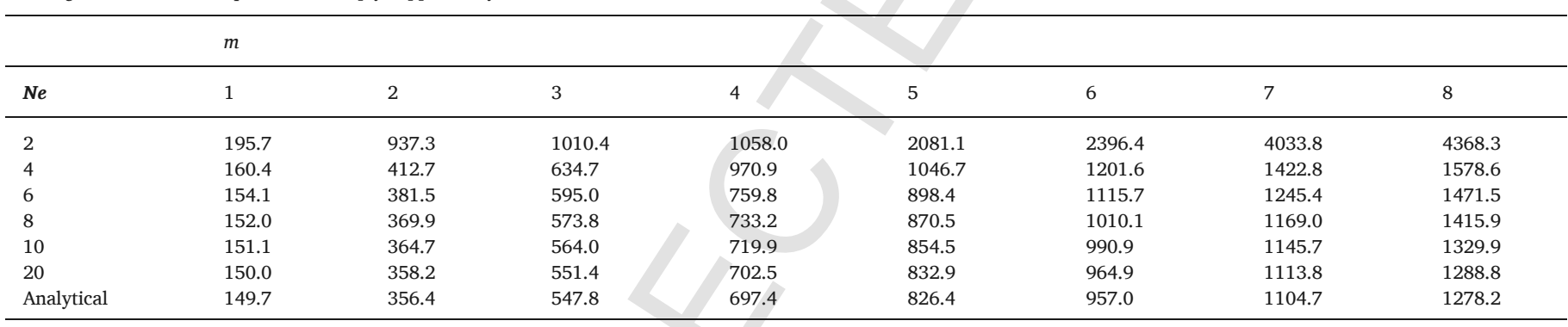

ness with the increase in the geometric stiffness due to the tensioning effect caused by pressurisation.

\subsection{Free shell}

Free vibration analysis of the cylindrical shell having the same geometric and material properties as in the previous example is performed for the case of free boundary conditions at the two ends of the shell. Natural frequencies calculated using 20 equal finite strips are listed in Table 6 . They are compared with the analytically determined values according to the procedure presented in [39] and summarised in Section 3.2. Small discrepancies can be noticed only for the higher axial mode profiles. Parameter $m$ denotes the number of vibration nodes of the axial mode profile. Some of the natural modes corresponding to the first 3 $\times 3 m \times n$ combinations are shown in Fig. 5 .

Some characteristic natural modes are shown in Fig. 6. They are identical to the analytically determined modes [39]. Natural modes with $m=0$ and $m=1$ are known as the Rayleigh-type and Love-type mode, respectively, following the nomenclature suggested in [11]. It is interesting that both symmetric and antisymmetric axial mode profiles are curved at the ends. The curvature is increased by increasing the wave number $n$ in a circumferential direction [39]. This phenomenon manifests the effect of the boundary conditions. A more detailed analysis of the mode shapes of free-free shells can be found in [35,39-42].

\subsection{Clamped shell}

A clamped steel cylindrical shell with the following geometric particulars and physical properties is considered next:

$$
\begin{aligned}
L & =2 \mathrm{~m}, a \\
& =1 \mathrm{~m}, \mathrm{~h} \\
& =0.01 \mathrm{~m}, E \\
& =2.1 \times 10^{11} \mathrm{~N} / \mathrm{m}^{2}, \quad v \\
& =0.3, \rho \\
& =7850 \mathrm{~kg} / \mathrm{m}^{3} .
\end{aligned}
$$

The values of the first 9 natural frequencies are listed in Table 7 and are compared with those determined by FEM [58]. Discrepancies

$\varepsilon(\%)=\frac{\omega_{F S M}-\omega_{F E M}}{\omega_{F E M}} \cdot 100$

show very good agreement of the natural frequencies determined in 

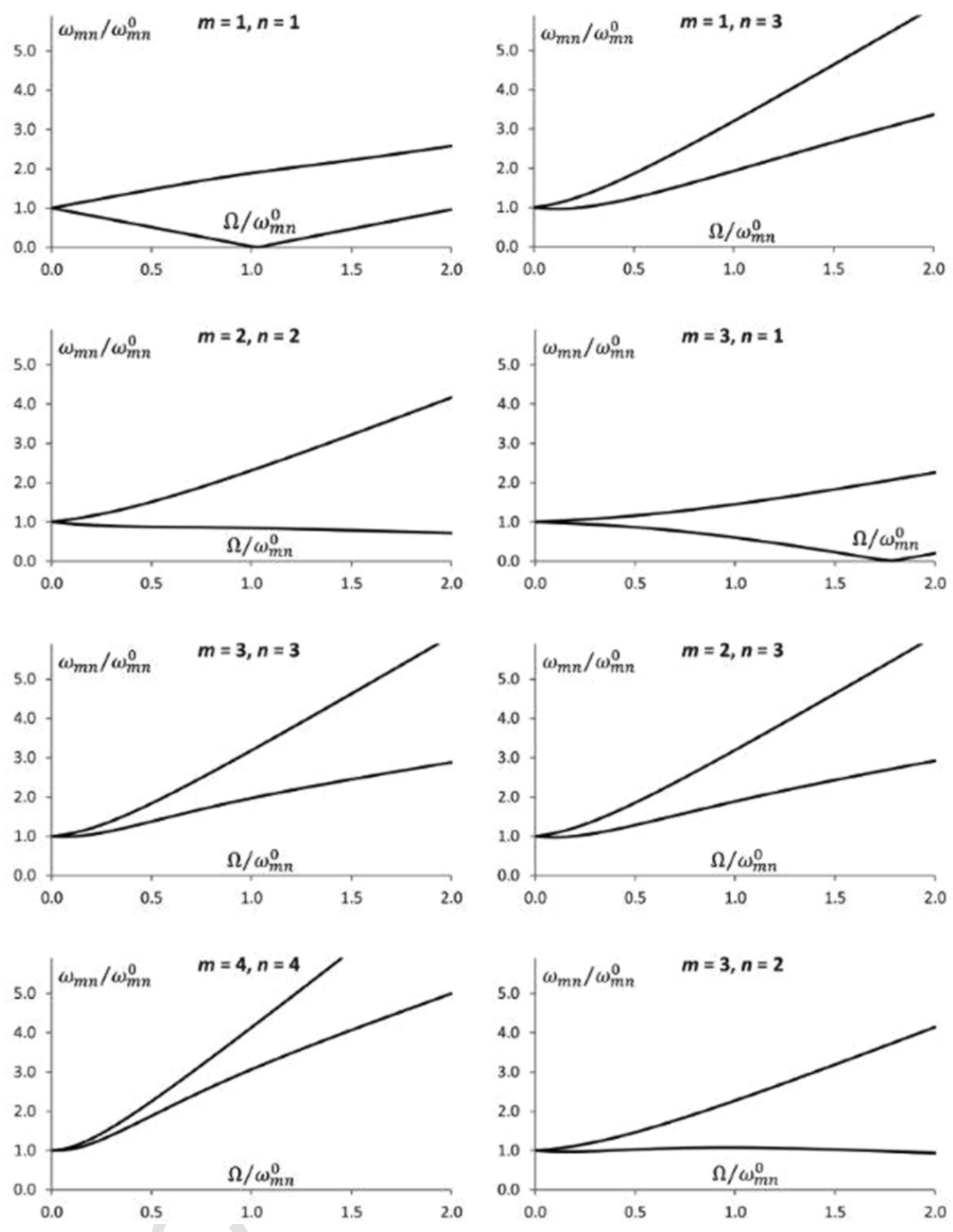

Fig. 3. Bifurcation of normalised natural frequencies of a rotating simply supported cylindrical shell.

two different ways. The corresponding natural modes are shown in Fig. 7 in an axonometric view.

\section{Conclusion}

Considering the vibration analysis of rotating cylindrical shells with arbitrary boundary conditions, there exist a number of analytical solutions in the relevant literature, but they are all rather complicated. The use of analytical procedures to model more complex shell structures would be rather involved. Therefore, special waveguide shell finite elements were developed in the past. However, none of the existing wave- guide shell finite elements are able to take into account the rotation effects or the initial stress effects (i.e. pressurisation).

In this paper, a new two node finite strip with eight degrees of freedom for the free vibration analysis of rotating and pre-stressed cylindrical shells is formulated. In order to develop the stiffness and mass matrices of the finite strip, simple bar and beam shape functions are used. The element stiffness matrix is shown to be a sum of an ordinary stiffness matrix and a geometric stiffness matrix. The geometric stiffness matrix is due to the initial tension forces. On the other hand, the inertia matrix is composed of three mass matrices. The first one is the ordinary mass matrix related to squared natural frequencies (inertia load), the second one is related to the squared angular velocity (centrifugal 


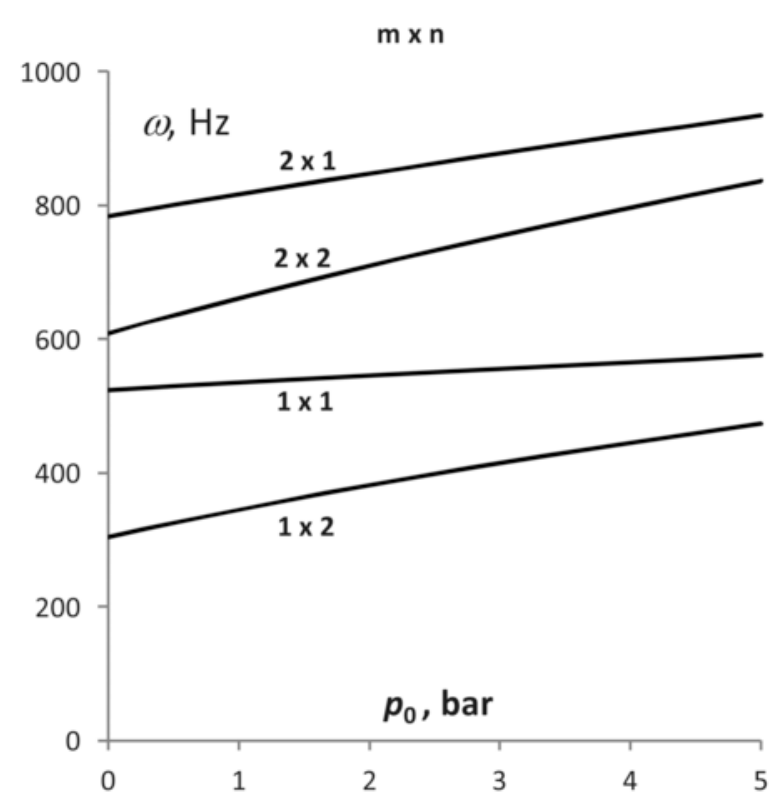

Fig. 4. Increase of natural frequencies of a simply supported cylindrical shell due to internal pressure.

load), and the third mass matrix is related to the multiple of a natural frequency and angular velocity (Coriolis load). The stiffness and mass matrices are composed of eleven submatrices which are effectively spatial integrals of products of different combinations of the shape functions and their derivatives. These integrals were calculated exactly and are given in Appendix A such that the proposed finite strip method can be used without further numerical burden.
The application of the developed finite strip is illustrated by numerical examples considering different boundary conditions at the two ends of the shell: simply supported, free, and clamped. The reliability of the finite strip was evaluated by an accuracy analysis and convergence analysis. A parametric analysis was also performed in order to appreciate the effects of the angular velocity and pre-stress on natural frequencies. It can be seen that the veering of the natural frequencies due to the Coriolis effect, as well as their upward shift due to pressurisation and the centrifugal effect, can be very accurately simulated.

Nowadays, the vibrations of rotating tyres are being extensively investigated primarily due to their importance in the Noise and Vibration Harshness (NVH) of a road vehicle. The cylindrical finite strip developed in this paper can be used to model the belt of a rotating tyre. In order to model a tyre sidewall, a conical of a toroidal finite strip would be necessary. In order to reduce the number of finite strips necessary to accurately model a shell structure, a sophisticated finite strip with an increased number of nodes and possibly faster convergence may also be considered.

Another problem related to the designing of shells of revolution is the forced vibrations and resonance phenomenon. Forced vibrations are widely analysed by the mode superposition method. Concerning cylindrical shells, they can be stationary with load travel in a circumferential direction [12]. Another interesting problem is the rotating cylindrical shell with a stationary dynamic load. Such an effect exists for an automobile tyre rolling on a smooth surface, [12]. In both cases, it is interesting to determine the critical load angular speed and the shell rolling speed, respectively, which equal the natural frequency of the structure. This challenging problem, in which the structure dynamic response is significantly increased, is a subject for further investigation.

Table 6

Natural frequencies of free cylindrical shell, $\omega[\mathrm{Hz}], \Omega=0, p_{0}=0$.

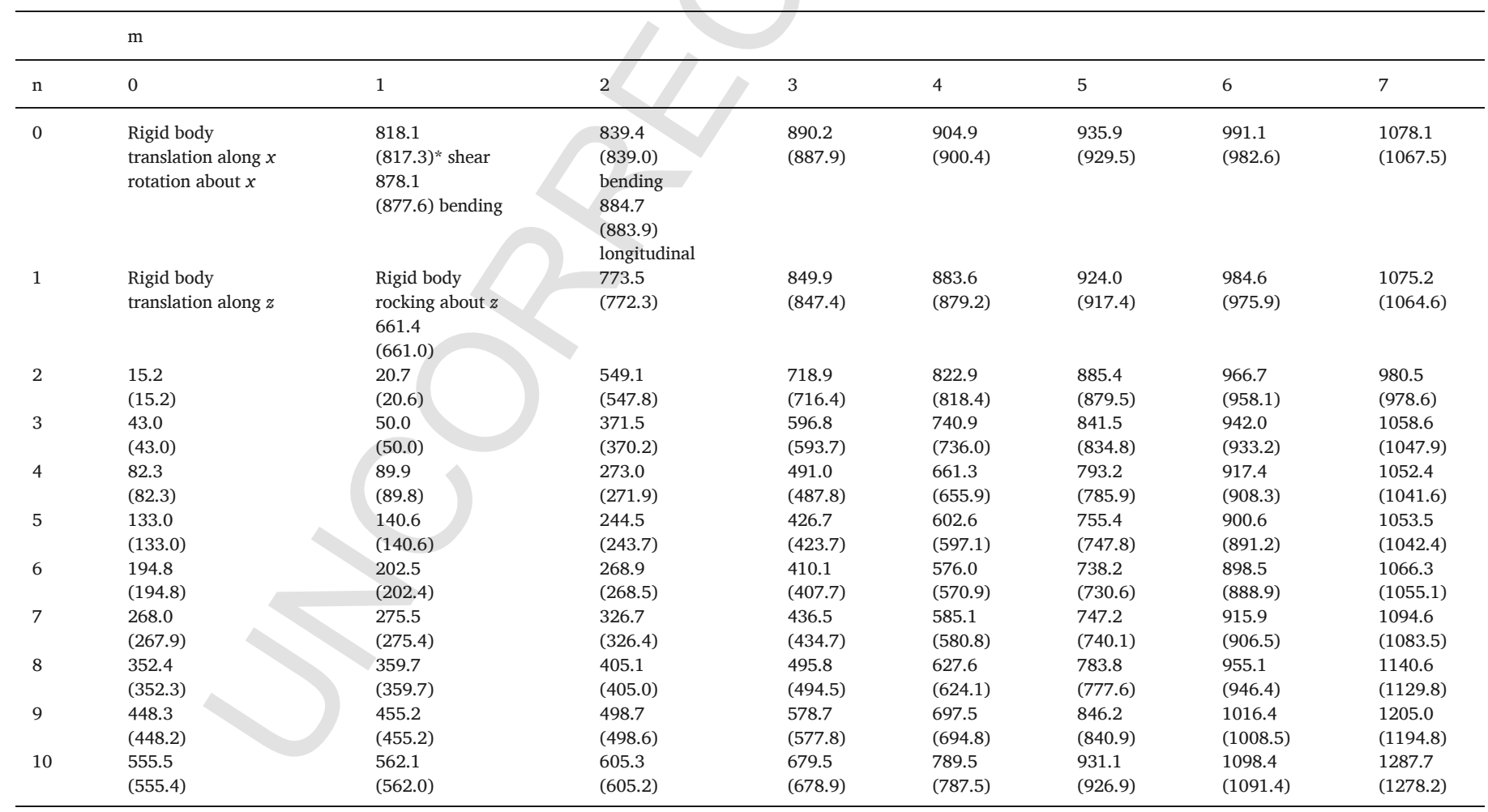

()* Analytical solution 


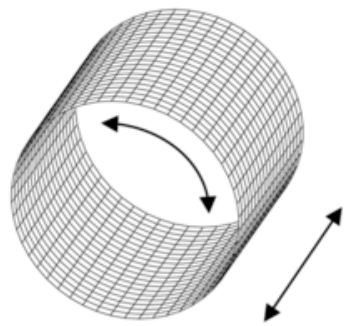

$(0,0)$

rigid body translation along $x$

$\&$ rotation about $x$

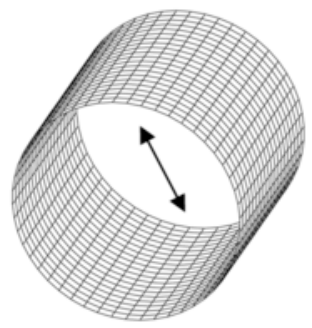

$(0,1)$

rigid body radial translation

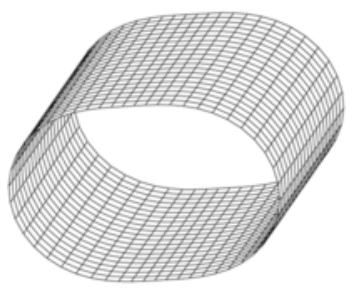

$(0,2)$

Rayleigh mode (bending) 15.2

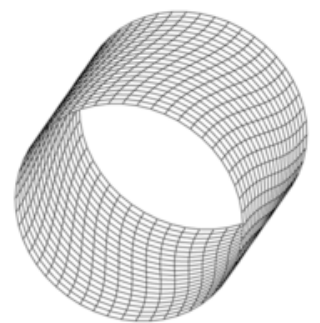

$(1,0)$

Love mode (shear) $817.3 \mathrm{~Hz}$

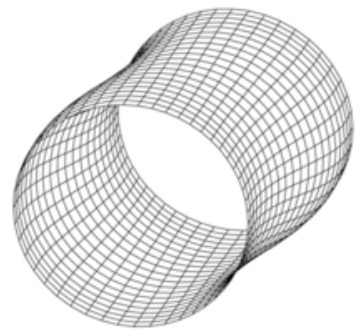

$(1,1)$

Love mode (bending) $661 \mathrm{~Hz}$

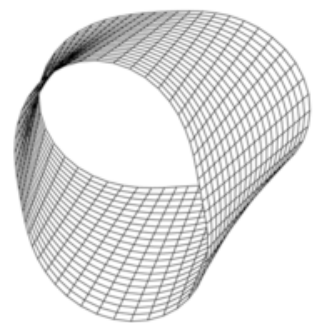

$(1,2)$

Love mode (bending) $20.7 \mathrm{~Hz}$

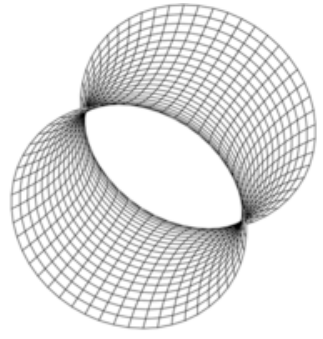

$(2,0)$

bending $839.0 \mathrm{~Hz}$

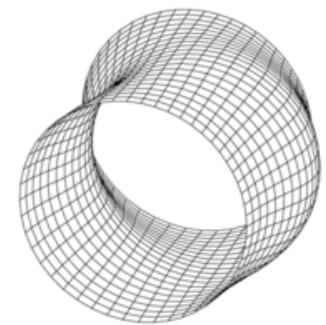

$(2,1)$

bending $772.3 \mathrm{~Hz}$

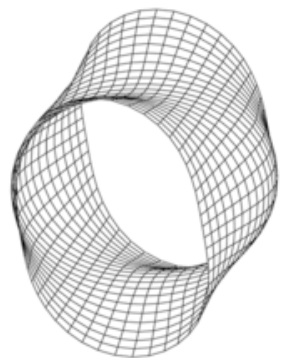

$(2,2)$

bending $547.7 \mathrm{~Hz}$

Fig. 5. Natural modes of the free cylindrical shell $(m, n)$.

\section{Uncited reference}

[32].

\section{Acknowledgements}

This project has received funding from the European Union's Horizon 2020 research and innovation programme under the Marie Sklodowska-Curie grant agreement no. 657539. This investigation also received funding within the international collaborative project Global Core Research Center for Ships and Offshore Plants (GCRC SOP), established by the South Korean Government (MSIP) through the National Research Foundation of South Korea (NRF).
Appendix A. Submatrices of the stiffness and mass matrices

$$
\begin{aligned}
& {[K]_{1}=\int_{0}^{l}\left[g^{\prime}{ }_{i} g_{j}{ }_{j}\right] \mathrm{d} x=\frac{1}{l}\left[\begin{array}{cc}
1 & -1 \\
-1 & 1
\end{array}\right]} \\
& {[K]_{2}=\int_{0}^{l}\left[g_{i} g_{j}\right] \mathrm{d} x=\frac{l}{6}\left[\begin{array}{cc}
2 & 1 \\
1 & 2
\end{array}\right]} \\
& {[K]_{3}=\int_{0}^{l}\left[{g^{\prime}}_{i} g_{j}\right] \mathrm{d} x=\frac{1}{2}\left[\begin{array}{cc}
-1 & -1 \\
1 & 1
\end{array}\right]}
\end{aligned}
$$



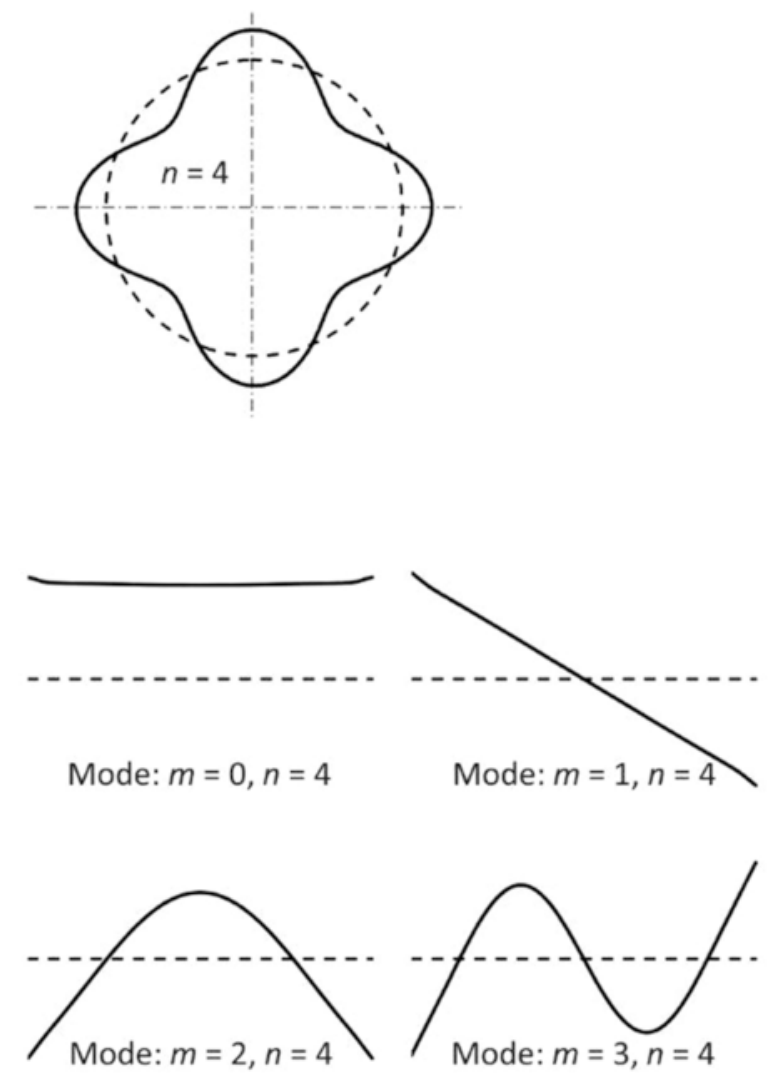

Fig. 6. Natural modes of the free cylindrical shell: circumferential and axial mode profiles, $\Omega=0, p_{0}=0$.

$$
\begin{aligned}
{[K]_{4} } & =\int_{0}^{l}\left[f^{\prime \prime}{ }_{i} f^{\prime \prime}{ }_{j}\right] \mathrm{d} x \\
& =\frac{2}{l^{3}}\left[\begin{array}{cccc}
6 & 3 l & -6 & 3 l \\
3 l & 2 l^{2} & -3 l & l^{2} \\
-6 & -3 l & 6 & -3 l \\
3 l & l^{2} & -3 l & 2 l^{2}
\end{array}\right]
\end{aligned}
$$$$
[K]_{5}=\int_{0}^{l}\left[f^{\prime}{ }_{i} f^{\prime}{ }_{j}\right] \mathrm{d} x
$$$$
=\frac{1}{30 l}\left[\begin{array}{cccc}
36 & 3 l & -36 & 3 l \\
3 l & 4 l^{2} & -3 l & -l^{2} \\
-36 & -3 l & 36 & -3 l \\
3 l & -l^{2} & -3 l & 4 l^{2}
\end{array}\right]
$$

$$
=\frac{l}{420}\left[\begin{array}{cccc}
156 & 22 l & 54 & -13 l \\
22 l & 4 l^{2} & 13 l & -3 l^{2} \\
54 & 13 l & 156 & -22 l \\
-13 l & -3 l^{2} & -22 l & 4 l^{2}
\end{array}\right]
$$

$[K]_{7}=\int_{0}^{l}\left[f^{\prime \prime} f_{j}+f_{i} f^{\prime \prime}{ }_{j}\right] \mathrm{d} x$

$$
=\frac{1}{15 l}\left[\begin{array}{cccc}
-36 & -18 l & 36 & -3 l \\
-18 l & -4 l^{2} & 3 l & l^{2} \\
36 & 3 l & -36 & 18 l \\
-3 l & l^{2} & 18 l & -4 l^{2}
\end{array}\right]
$$

$[K]_{8}=\int_{0}^{l}\left[g^{\prime} f_{j}\right] \mathrm{d} x=\frac{1}{12}\left[\begin{array}{cccc}-6 & -l & -6 & l \\ 6 & l & 6 & -l\end{array}\right]$

$[K]_{9}=\int_{0}^{l}\left[g^{\prime}{ }_{i} f_{j}^{\prime}\right] \mathrm{d} x=\frac{1}{l}\left[\begin{array}{cccc}1 & 0 & -1 & 0 \\ -1 & 0 & 1 & 0\end{array}\right]$

$[K]_{10}=\int_{0}^{l}\left[g_{i} f_{j}\right] \mathrm{d} x=\frac{l}{60}\left[\begin{array}{cccc}21 & 3 l & 9 & -2 l \\ 9 & 2 l & 21 & -3 l\end{array}\right]$

$[K]_{11}=\int_{0}^{l}\left[g_{i} f^{\prime \prime}{ }_{j}\right] \mathrm{d} x=\frac{1}{l}\left[\begin{array}{cccc}-1 & -l & 1 & 0 \\ 1 & 0 & -1 & l\end{array}\right]$

It is worth mentioning that some of the above submatrices have the following physical meanings:

\begin{tabular}{|c|c|c|c|c|c|c|c|c|c|}
\hline Mode no. & 1 & 2 & 3 & 4 & 5 & 6 & 7 & 8 & 9 \\
\hline$n$ & 6 & 5 & 7 & 4 & 8 & 9 & 7 & 3 & 8 \\
\hline FSM & 133.0 & 139.1 & 146.3 & 168.6 & 173.9 & 211.3 & 226.1 & 226.2 & 233.1 \\
\hline FEM & 132.1 & 138.2 & 145.5 & 167.8 & 173.0 & 210.2 & 223.0 & 225.6 & 229.7 \\
\hline$\varepsilon(\%)$ & 0.68 & 0.62 & 0.62 & 0.46 & 0.54 & 0.48 & 1.39 & 0.30 & 1.47 \\
\hline
\end{tabular}

$E A[K]_{1}$ - bar stiffness matrix,

Table 7

Natural frequencies of clamped cylindrical shell, $\omega[\mathrm{Hz}], \Omega=0, p_{0}=0$. 

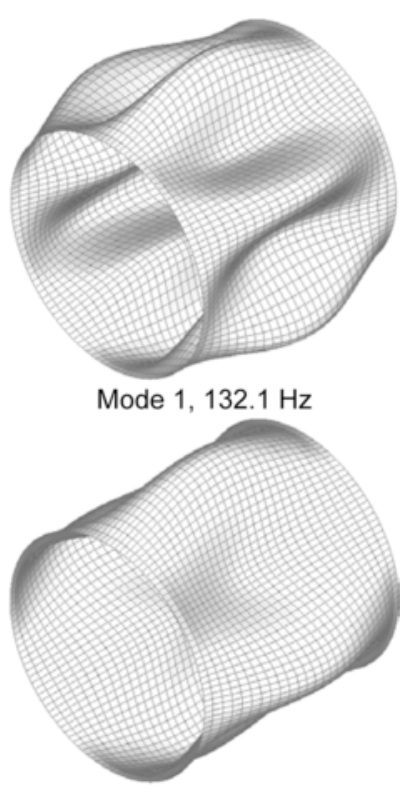

Mode 4, $167.8 \mathrm{~Hz}$

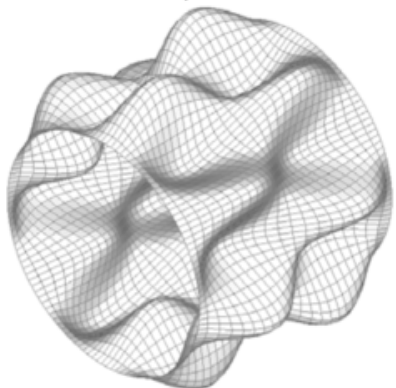

Mode 7, 223.0 Hz

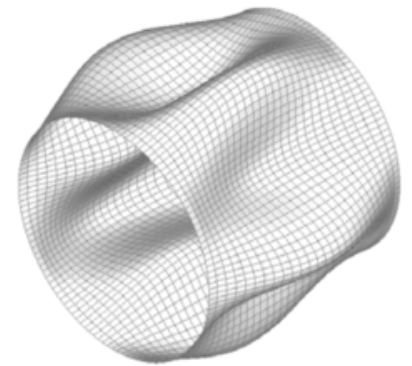

Mode 2, $138.2 \mathrm{~Hz}$

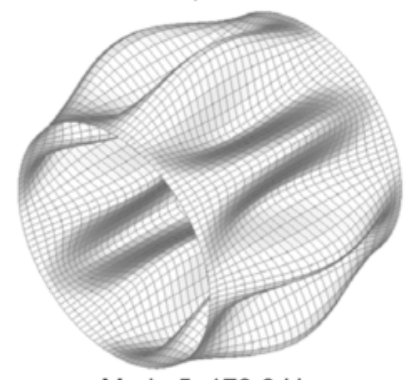

Mode $5,173.0 \mathrm{~Hz}$

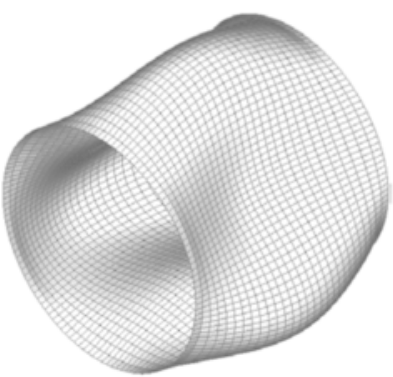

Mode $8,225.5 \mathrm{~Hz}$
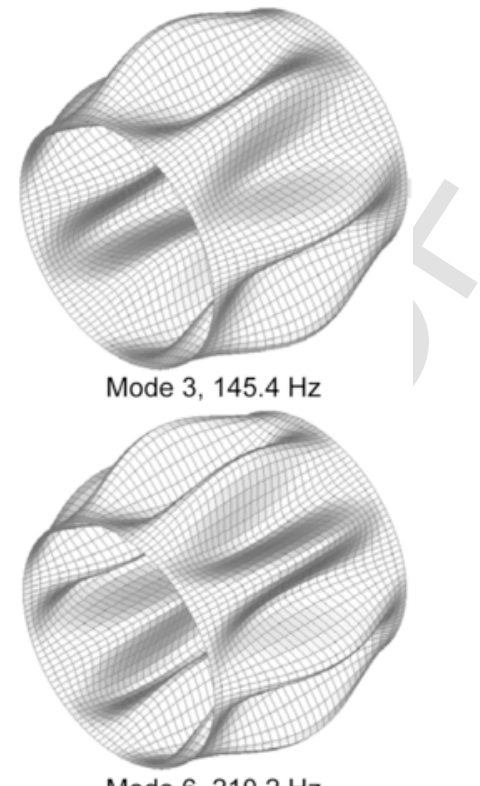

Mode 6, 210.2 Hz

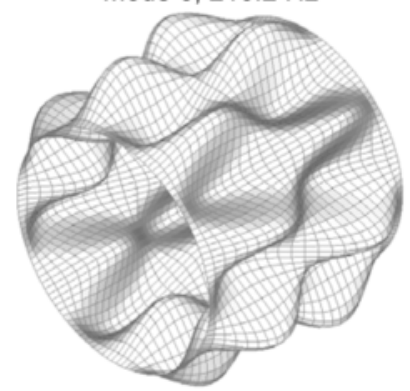

Mode 9, 229.7 Hz

Fig. 7. Natural modes of the clamped cylindrical shell, $\Omega=0, p_{0}=0$.

$\rho A[K]_{2}$ - bar mass matrix,

$E I[K]_{4}$ - beam stiffness matrix,

$N[K]_{5}$ - beam geometric stiffness matrix,

$k[K]_{6}$ - beam restoring stiffness matrix,

$\rho A[K]_{6}$ - beam mass matrix.

Symbols $A$ and $I$ denote a cross-sectional area and its moment of inertia, respectively, $N$ is an axial force and $k$ is the stiffness of an elastic foundation. Submatrix $[K]_{6}$ appears in both the stiffness and the mass matrix Eqs. (37) and (45).

\section{Appendix B. Geometric stiffness matrix}

The general stiffness matrix $[K]$, Eq. (37), consists of: a) ordinary stiffness matrix $[K]_{0}$ without the initial tension forces, $N_{x}=N_{?}=0$, Eq. (4); b) ordinary geometric stiffness matrix $[K]_{G p}$ with tension forces due to the internal pressure $N_{x}=a p_{0} / 2$ and $N_{?}=a p_{0}$, Eq. (4); and c) the specific geometric stiffness matrix $[K]_{G \Omega}$ due to the centrifugal force induced by the angular velocity. The general stiffness matrix is $[K]=[K]_{0}+p[K]_{G p}+\Omega^{2}[K]_{G \Omega}$. Both geometric stiffness matrices increase natural frequencies.

The $[K]_{G p}$ matrix is interesting for buckling the stability analysis. It can be expressed in the form:

$$
\begin{aligned}
& {[K]_{G p}} \\
& =\left[\begin{array}{ccc}
c_{1}[K]_{1}+c_{2}[K]_{2} & {[0]} & {[0]} \\
{[0]} & c_{3}[K]_{1}+c_{4}[K]_{2} & d_{1}[K]_{10} \\
{[0]} & d_{1}[K]_{10}^{T} & d_{2}[K]_{5}+d_{3}[K]_{6}
\end{array}\right],
\end{aligned}
$$

where

$c_{1}=\frac{1}{2} \pi a^{2}, \quad c_{2}=\pi n^{2}, \quad c_{3}=\frac{1}{2} \pi a^{2}, \quad c_{4}=\pi$, $d_{1}=2 \pi n, \quad d_{2}=\frac{1}{2} \pi a^{2}, \quad d_{3}=\pi\left(1+n^{2}\right)$.

The finite strip equation for the stability analysis reads

$$
\{F\}=\left([K]_{0}-p_{c r}[K]_{G}\right)\{\delta\},
$$

where $p_{c r}$ is the critical external pressure which causes buckling. The linear stability analysis can be performed in an ordinary way through the finite element method, leading to an eigenvalue formulation for the determination of the critical pressure.

\section{References}

[1] H. Aron, Das Gleichgewicht und die Bewegung einer unendlich dünnen, beliebig gekrümmten elastischen Schale. (The equilibrium and the motion of an infinitely thin, arbitrarily curved elastic shell), J. Math. (Crelle) 78 (1874).

[2] A.E.H. Love, On the small free vibrations and deformations of thin elastic shells, Philos. Trans. R. Soc. Lond. 179A (1888). 
[3] J.W.S. Rayleigh, On the Infinitesimal Bending of Surfaces of Revolution, Math. Soc. Proc. 13, London, 1882.

[4] S. Timoshenko, S. Woinowsky-Krieger, Theory of Plates and Shells, McGraw-Hill Book Company, New York, 1959.

[5] V.V. Novozhilov, The Theory of Thin Elastic Shells, P. Noordhoff, Gromingen, The Netherlands, 1964.

[6] W. Flügge, Statik and Dynamik der Schalen (Statics and Dynamics of Shells), Springer-Verlag, Berlin, 1934

[7] A.L. Goldenveizer, Theory of Thin Shells, Pergamon Press, Elmsford, New York, 1961.

[8] V.Z. Vlasov, General Theory of Shells and Its Applications in Engineering (translation from Russian), NASA TTF-99, U.S. Governmental Printing Office, Washington, D.C, 1964.

[9] J.E. Gibson, Linear Elastic Theory of Thin Shells, Pergamon Press, London, 1965

[10] L.H. Donnell, Beams, Plates, and Shells, McGraw-Hill, New York, 1976.

[11] A.W. Leissa, Vibrations of Shells, NASA SP-288, U.S. Government Printing Office, Washington, D.C., 1973.

[12] W. Soedel, Vibrations of Shells and Plates 3rd edition, Marcel Dekker Inc, New York, 2004(revised and expanded).

[13] C.T.F. Ross, Pressure Vessels Under External Pressure: Statics and Dynamics, Elsevier Applied Science, London, 1990.

[14] Group of Authors, in E. E. Allmenidinger, (Ed.), Submersible Vehicle System Design, SNAME, Jersey City, 1990.

[15] I. Senjanović, Theory of Shells of Revolution, Ship Research Institute, Zagreb, 1972.

[16] G.H. Bryan, On the Beats in the Vibrations of a Revolving Cylinder or Bell. In: Proc. of the Camb. Philos. Soc. 101-111, 1880.

[17] R.A. Di Taranto, M. Lessen, Coriolis acceleration Effect on the vibration of a rotating thin-walled circular cylinder, J. Appl. Mech. 31 (1964) 700-701.

[18] A.V. Srinivasan, G.F. Lauterbach, Traveling waves in rotating cylindrical shells, J. Eng. Ind. ASME 93 (1971) 1229-1232.

[19] A. Zohar, J. Aboudi, The free vibrations of a thin circular finite rotating cylinder, Int. J. Mech. Sci. 15 (1973) 269-278.

[20] T. Saito, M. Endo, Vibration of finite length, rotating cylindrical shells, J. Sound Vib. 107 (1986) 17-28

[21] M. Endo, K. Hatamura, M. Sakata, O. Taniguchi, Flexural vibration of a thin rotating ring, J. Sound Vib. 92 (1984) 261-272.

[22] J. Padovan, Natural frequencies of rotating prestressed cylinders, J. Sound Vib. 31 (1973) 469-482.

[23] G. Herrmann, A.E. Armenakas, Dynamic Behavior of Cylindrical Shells under Initial Stress, Proc. 4th U.S. Nat. Congr. Appl. Mech, ASME, 203-213, 1962.

[24] S.C. Huang, W. Soedel, On the forced vibration of simply supported rotating cylindrical shells, J. Acoust. Soc. Am. 84 (1) (1988) 275-285.

[25] C. Gonzalez Diaz, P. Kindt, J. Middelberg, S. Vercammen, C. Thiry, R. Close, J. Leyssens, Dynamic behaviour of a rolling tyre: experimental and numerical analyses, J. Sound Vib. 364 (2016) 147-164.

[26] J. Lee, S. Wang, P. Kindt, B. Pluymers, W. Desmet, Identification of the direction and value of the wave length of each mode for a rotating tire using the phase difference method, Mech. Syst. Signal Process. 68-69 (2016) 292-301.

[27] P. Kindt, C.G. Diaz, S. Vercammen, C. Thiry, J. Middelberg, B. Kimble, J. Leyssens, Effects of rotation on the tire dynamic behavior: experimental and numerical analyses, Tire Sci. Technol. 41 (4) (2013) 248-261.

[28] W.R. Graham, Modelling the vibration of tyre sidewalls, J. Sound Vib. 332 (21) (2013) 5345-5374.

[29] C. Lecomte, W.R. Graham, M. Dale, A shell model for tyre belt vibrations, J. Sound Vib. 329 (10) (2010) 1717-1742.

[30] Y.-J. Kim, J.S. Bolton, Effects of rotation on the dynamics of a circular cylindrical shell with application to tire vibration, J. Sound Vib. 275 (2004) 605-621.

[31] L.R. Molisani, R.A. Burdisso, D. Tsihlas, A coupled tire structure/acoustic cavity model, Int. J. Solids Struct. 40 (2003) 5125-5138.

[32] V.Z. Vlasov, Basic Differential Equations in the General Theory of Elastic Shells, NACA TM 1241 (translated from 1944 Russian version), 1951.

[33] K. Forsberg, Influence of boundary conditions on the modal characteristics of thin cylindrical shells, AIAA J. 2 (12) (1964) 2150-2157.
[34] V.I. Weingarten, On the free vibration of thin cylindrical shells, Aerospace corporation, Systems research and planning division, Report No. TDR.169(3560.30)TN-3, El Segundo, California, 1962.

[35] G.B. Warburton, Vibration of thin cylindrical shells, J. Mech. Eng. Sci. 7 (4) (1965) 399-407.

[36] H. Chung, Free vibration analysis of circular cylindrical shells, J. Sound Vib. 74 (1981) 331-350.

[37] S. Sun, S. Chu, D. Cao, Vibration characteristics of thin rotating cylindrical shells with various boundary conditions, J. Sound Vib. 331 (2012) 4170-4186.

[38] S. Sun, D. Cao, Q. Han, Vibration studies of rotating cylindrical shells with arbitrary edges using characteristic orthogonal polynomials in the Rayleigh-Ritz method, Int. J. Mech. Sci. 68 (2013) 180-189.

[39] N. Alujević, N. Campillo-Davo, P. Kindt, W. Desmet, B. Pluymers, S. Vercammen, Analytical solution for free vibrations of rotating cylindrical shells having free boundary conditions, Eng. Struct. 132 (2017) 152-171.

[40] N. Alujević, N. Campillo-Davo, P. Kindt, W. Desmet, B. Pluymers, S.Vercammen, A simplified tire model based on a rotating shell, Proceedings of the 4th International Tyre Colloquium, University of Surrey, Surrey, 2015.

[41] N. Alujevic, N. Campillo-Davo, P. Kindt, W. Desmet, B. Pluymers, S. Vercammen, A simplified model of a rotating tire using cylindrical shells with free ends supported by an elastic foundation, Proceedings of ISMA2014, Katholieke Universiteit Leuven, Leuven, 2014

[42] N. Alujevic, P. Kindt, B. Pluymers, P. Sas, W. Desmet, N. Campillo-Davo, Simplified rotating tire models based on cylindrical shells with free boundary conditions, Proceedings of FISITA 2014, World Automotive Congress, 30 Percy Street, London, art.nr. F2014-NVH-084, 2014.

[43] S. Finnreden, M. Fraggstedt, Waveguide finite element for curved structures, J. Sound Vib. 312 (2008) 644-671

[44] P. Sabiniarz, W. Kropp, A waveguide finite element aided analysis of the wave field on a stationary tyre, not in contact with the ground, J. Sound Vib. 329 (2010) 3041-3064.

[45] J.M. Renno, B.R. Mace, Calculating the forced response of cylinders and cylindrical shells using the wave and finite element method, J. Sound Vib. 333 (2014) 5340-5355.

[46] Y.K. Cheung, Finite Strip Method in Structural Analysis, Pergamon Press, Oxford, 1976.

[47] J.G. Hancock, C.H. Pham, Buckling analysis of thin-walled sections under localised loading using the semi-analytical finite strip method, Thin-Walled Struct. 86 (2015) 35-46.

[48] H.R. Naderian, H.R. Ronagh, Buckling analysis of thin-walled cold-formed stee structural members using complex finite strip method, Thin-Walled Struct. 90 (2015) 74-83.

[49] A. Naghsh, M.M. Saadatpour, M. Azhari, Free vibration analysis of stringer stiffened general shells of revolution using a meridional finite strip method, Thin-Walled Struct. 94 (2015) 651-662.

[50] H. Assaee, H. Hasani, Forced vibration analysis of composite cylindrical shells using spline finite strip method, Thin-Walled Struct. 97 (2015) 207-214.

[51] J.C.G. Verschaeve, A weighted extended B-spline solver for bending and buckling of stiffened plates, Thin-Walled Struct. 107 (2016) 580-596.

[52] M. Khezri, M. Abbasi, K.J.R. Rasmussen, A combined meshfree/finite strip method for analysis of plates with perforations and cracks, Thin-Walled Struct. 111 (2017) $113-125$.

[53] S.S. Ajeesh, S.A. Jayachandran, A constrained spline finite strip method for the mode decomposition of cold-formed steel sections using GBT principles, Thin-Walled Struct. 113 (2017) 83-93.

[54] M.A. Rendall, G.J. Hancock, K.J.R. Rasmussen, The generalised constrained finite strip method for thin-walled members in shear, Thin-Walled Struct. 117 (2017) 294-302.

[55] A. Borkovic, S. Kovacevic, D.D. Milasinovic, G. Radenkovic, O. Mijatovic, V. Golubovic-Bugarski, Geometric nonlinear analysis of prismatic shells using the semi-analytical finite strip method, Thin-Walled Struct. 117 (2017) 63-88.

[56] A.E.H. Love, A Treatise on the Mathematical Theory of Elasticity, 4th ed, Dover, New York, 1927.

[57] R. Szilard, Theories and Applications of Plate Analysis, John Wiley \& Sons, 2004

[58] MSC NASTRAN Installation and Operations Guide MSC Software, MSC 2005 